\title{
SIMMETRIE SPEZZATE. NATURA, NASCITA ED EVOLUZIONE DELLA PROPRIETÀ PRIVATA
}

\author{
ANTONIO ZANELLA*
}

\begin{abstract}
Il liberalismo, fin dalle sue origini, si basa sul riconoscimento e sul rispetto di tre diritti fondamentali: vita, proprietà e libertà —uno dei fondatori di questa dottrina politica fu il filosofo inglese John Locke (16321704)—. Per il liberalismo classico la libertà e la proprietà sono strettamente correlate; i liberali e, in seguito, i libertari, si sono spinti oltre affermando che la libertà e la proprietà sono la stessa cosa. Anche il diritto alla vita è stato riformulato nei termini di proprietà di sé, cioè ognuno è proprietario del proprio corpo e del proprio intelletto, insomma della propria vita. I tre diritti fondamentali —inalienabili_ che stanno alla base di ogni altro diritto sono quindi riconducibili al diritto di proprietà. Nel giustificare questo diritto alcuni autori liberali e libertari — come per esempio Murray N. Rothbard (19261995) - si sono appoggiati al giusnaturalismo, cioè a una dottrina filosoficogiuridica che si basa sul riconoscimento dell'esistenza di un diritto naturale e razionale universalmente valido, considerato il fondamento di ogni diritto civile. In questo lavoro cercheremo di giustificare il concetto di proprietà abbandonando la sua fondazione giusnaturalistica, che molti contestano per le sue pretese universalistiche - non tutti, infatti, sono disposti a riconoscere I'universalismo dei diritti naturali e l'aura di "sacralità» e "misticismo" che sembrano emanare- sforzandoci di dare un fondamento razionale al rispetto che gli individui tendono ad avere nei confronti dei diritti di proprietà. Per fare ciò utilizzeremo la branca della teoria della scelta razionale che si occupa delle decisioni interattive o strategiche: la teoria dei giochi.

In questo passo mostreremo, quindi, servendoci dei principi della teoria evoluzionistica dei giochi (TEG), ' come il concetto di proprietà privata non sia arbitrario, ma sia nato e si sia evoluto per la sua efficienza nel dirimere contese, sia nel mondo animale che tra gli esseri umani. Non sono solo gli esseri umani, infatti, a riconoscere e rispettare la proprietà altrui; anche la maggior parte degli animali distinguono tra lo stato di proprietario e quello
\end{abstract}

\footnotetext{
* Universidad Rey Juan Carlos.

${ }^{1}$ Un'interessante introduzione alla TEG si trova in Weibull (1997).
} 
di intruso e si comportano in modo diverso qualora si trovino nel primo o nel secondo.

Nel primo paragrafo forniremo una breve introduzione ai concetti fondamentali della teoria evoluzionistica dei giochi; nel secondo definiremo il concetto di proprietà privata. Nel terzo considereremo, sulle tracce di David Friedman e degli autori della scuola "austriaca», ${ }^{2}$ la funzione sociale della proprietà privata, per passare poi, nel quarto paragrafo, all'analisi della nascita e dell'evoluzione della proprietà, analisi che verrà condotta sulla base della TEG.

\author{
"La proprietà è il diritto reale che attribuisce \\ al proprietario la facoltà di godere e disporre \\ della cosa in modo pieno ed esclusivo, \\ entro i limiti e con l'osservanza degli obblighi \\ stabiliti dall'ordinamento giuridico \\ (C. Bianca, G. Patti e S. Patti, \\ Lessico di Diritto Civile, 2001, 832 cc)» \\ "L'origine della proprietà giace nelle \\ simmetrie spezzate \\ (Skyrms, 1996, p. 79)»
}

\title{
LA TEORIA EVOLUZIONISTICA DEI GIOCHI
}

La teoria evoluzionistica dei giochi - d'ora in poi TEG- è stata sviluppata a partire dai primi anni settanta dal biologo John Maynard Smith e da alcuni suoi colleghi. ${ }^{3}$ Questa teoria combina le idee della teoria matematica, o classica, dei giochi con quelle della biologia evoluzionistica. ${ }^{4}$

${ }^{2}$ David Friedman —d'ora in poi D. Friedman —è figlio del noto economista Milton Friedman, autore, tra gli altri, di Capitalismo e libertà (1962), in cui difende il ruolo economico e politico della proprietà.

${ }^{3}$ Per una esposizione sintetica delle ricerche di Maynard Smith si veda: Evolution and the Theory of Games (1982). Un'interessante introduzione alla TEG si trova anche in Weibull (1997).

${ }^{4}$ Per la stesura di questo paragrafo ci siamo avvalsi dell'utilissima introduzione alla TEG di Festa (1999). 
«Un'assunzione fondamentale della biologia evoluzionistica è quella per cui la dinamica dell'evoluzione biologica si basa sulla riproduzione differenziale degli individui più adatti» (Festa, 1999, p. 2). La selezione naturale - che Charles Darwin applicava alle mutazioni genetiche - viene ora applicata, in TEG, anche alle regole di comportamento, cioè alle diverse strategie possibili all'interno di un determinato tipo di gioco. In tal modo si può spiegare la sopravvivenza delle regole più adatte. "Le principali caratteristiche qualitative della riproduzione differenziale vengono catturate da un semplice modello dinamico, sviluppato piuttosto recentemente, noto come dinamica dei riproduttori» (Festa, 1999, p. 2).

«La TEG si basa sull'idea di applicare la dinamica dei riproduttori a certe popolazioni di giocatori allo scopo di comprendere l'evoluzione di determinate strategie di gioco. Più precisamente, essa si basa sull'assunzione che esista qualche tipo di dinamica evoluzionistica che accresce la proporzione dei giocatori che utilizzano strategie che hanno dato buoni risultati in confronto alla media della popolazione» (Festa, 1999, p. 3).

Sono stati fatti alcuni esperimenti di simulazione computerizzata — per esempio, Axelrod (1984) — basati sui modelli dinamici della TEG. ${ }^{5}$ "Questi esperimenti consistono in una serie di tornei in seguito ai quali le strategie di maggior successo - cioè quelle che ottengono un payoff più alto- sono ammesse a partecipare a un numero maggiore di incontri, mentre viene limitato il grado di partecipazione delle strategie meno fortunate. Ogni torneo rappresenta una generazione di individui ciascuno dei quali impiega costantemente una determinata strategia nelle sue interazioni con altri membri della popolazione. Il successo di una strategia in ogni singolo torneo determinerà la numerosità della sua "prole", e quindi la frequenza relativa con cui tale strategia sarà rappresentata nella generazione successiva. A partire da una determinata distribuzione iniziale delle strategie nella popolazione, e del valore di alcuni altri parametri rilevanti, ${ }^{6}$ si può stabilire,

${ }^{5}$ Per un'applicazione delle simulazioni computerizzate allo studio della società si veda Epstein e Axtell (1996).

6 Tali parametri includono, per esempio, il parametro di sconto e la probabilità di ogni strategia di misurarsi con se stessa. 
attraverso ben congegnati esperimenti di simulazione, come evolveranno le diverse strategie e quale sarà la loro distribuzione finale nella popolazione» (Festa, 1999, pp. 10-11). Una grave limitazione degli esperimenti di simulazione di Axelrod (1984) è costituita dalla loro impostazione ecologica, cioè dalla circostanza che -mentre si consente che nelle generazioni successive possano variare le frequenze con cui le diverse strategie sono inizialmente rappresentate nella popolazione- non si prevede la possibilità di introdurre nuove strategie. L'evoluzione biologica, al contrario, è in grado di introdurre nuove strategie nella popolazione, attraverso la mutazione e altri meccanismi che forniscono fonti di variabilità per le strategie rappresentate nella popolazione.

Dopo il 1984 Axelrod ha sviluppato un metodo per generare automaticamente nuove strategie, senza alcuna progettazione umana. Il metodo da lui escogitato (Axelrod, 1987) è costituito da un'appropriata versione degli algoritmi genetici sviluppati da John Holland (1975). ${ }^{7}$ "Gli algoritmi genetici sono stati sviluppati con l'intento di riprodurre i meccanismi sui quali si basa il grande successo dell'evoluzione biologica nello scoprire metodi complessi ed efficaci per l'adattamento a una grande varietà di situazioni ambientali» (Festa, 1999, p. 12). L'evoluzione biologica, infatti, utilizza sia meccanismi genetici di riproduzione differenziale - che consentono alla prole di ereditare le caratteristiche o strategie più riuscite dei genitori- sia meccanismi per la scoperta di nuove caratteristiche o strategie. Questi ultimi includono la riproduzione sessuale, attraverso la quale la prole eredita parte del materiale genetico da un genitore e parte dall'altro, e la mutazione (Festa, 1999, p. 12). Gli algoritmi genetici devono il proprio nome al fatto di imitare la doppia funzione dei cromosomi nell'evoluzione biologica, più precisamente:

i) essi forniscono una rappresentazione di ciò che l'organismo diverrà;

7 Per un'esposizione di carattere introduttivo agli algoritmi genetici si vedano Holland (1992), Riolo (1992) e Mitchell (1996). 
ii) il loro materiale genetico è suscettibile di subire trasformazioni, così da trasmettere alla generazione successiva un «nuovo» materiale.

«A partire da una popolazione iniziale ottenuta pescando a caso da tutte le strategie possibili, un algoritmo genetico conduce, generazione dopo generazione, a popolazioni finali molto diverse da quella iniziale, non solo per la diversa frequenza delle strategie sopravvissute, ma anche per la comparsa di nuove strategie. Dopo molte generazioni si ottiene spesso, come risultato della simulazione, una popolazione composta da individui che, nel dato ambiente strategico, ottengono mediamente un successo maggiore di quelli della popolazione iniziale» (Festa, 1999, pp. 12-13).

Dati i notevoli successi ottenuti dalla TEG nella spiegazione del comportamento animale, la sua applicazione è stata estesa ad altri campi come l'evoluzione della cooperazione, ${ }^{8} \mathrm{l}^{\prime}$ evoluzione delle norme di giustizia, ${ }^{9}$ e l'analisi dell'evoluzione culturale. ${ }^{10}$

Un concetto fondamentale della TEG - introdotto da Maynard Smith (1982) - è quello di strategia evolutivamente stabile, che $\mathrm{d}^{\prime}$ ora in poi indicheremo con l'acronimo SES.

Quale strategia ha maggiori probabilità di successo in un contesto evolutivo? Innanzitutto deve essere una strategia che ottiene buoni risultati giocando contro se stessa; altrimenti, se la strategia invadesse la popolazione, cioè si diffondesse in tutta la popolazione, sarebbe indebolita a causa della sua mancanza di successo; in secondo luogo, deve essere capace di invadere una popolazione di strategie alternative e, a tal fine, deve comportarsi meglio nel caso di uno scontro diretto con altre strategie. Queste caratteristiche suggeriscono la seguente definizione di SES:

(SES) Strategia evolutivamente stabile. Una SES è una strategia che, in primo luogo, costituisce una risposta ottimale a se stessa; in secondo luogo, se una qualunque altra strategia ottiene contro una data SES lo stesso risultato della SES, allora la SES

\footnotetext{
8 Si veda, per esempio, Axelrod (1984).

9 Si veda Skyrms (1996).

10 cfr. Leinfellner (1998) e Selten (1998).
} 
deve ottenere, nel confronto con se stessa, un risultato migliore di quello ottenuto dall'altra strategia nel confronto con se stessa. ${ }^{11}$

\section{II}

\section{IL CONCETTO DI PROPRIETÀ PRIVATA}

Il termine «proprietà» indica le regole che governano l'accesso alle - e il controllo delle - risorse materiali, come la terra, e di quelle intellettuali, come le idee e le invenzioni. ${ }^{12}$ Più specificamente, l'espressione "proprietà privata» si riferisce a un sistema di distribuzione delle risorse che alloca particolari beni o oggetti - come appezzamenti di terreno- a particolari individui, affinché li usino secondo il loro volere, escludendo gli altri dal loro uso. La caratteristica principale del concetto di proprietà è l'esclusione. L'esclusione è la negazione, da parte del proprietario, dell'accesso di altri soggetti alle risorse da lui possedute senza il suo permesso.

È proprio il concetto di esclusione, alla base dell'idea di proprietà privata, che rende questa idea problematica. Il concetto di esclusione, di per sé, non è problematico, ma lo diventa se si considera il fatto che le risorse che si possiedono sono scarse e spesso necessarie, come nel caso del cibo, dell'acqua e così via. Se, infatti, un solo uomo possedesse tutte le riserve idriche del pianeta potrebbe decidere di non condividerle con nessuno e il rispetto dei diritti di proprietà imporrebbe a tutti gli altri abitanti del pianeta di morire di sete. D'altra parte, se le risorse fossero liberamente disponibili, cioè non possedute da alcun individuo, e non vi fossero regole che determinassero il loro uso, si creerebbero continui conflitti con i conseguenti sprechi di tempo, energia e beni. Ogni società interessata a evitare i conflitti connessi alla lotta per risorse scarse necessita di regole che determinino l'accesso a tali risorse; così da permetterne anche la produzione e lo scambio.

11 Vedi Hargreaves (1992, pp. 169-170).

12 Per la stesura di questo paragrafo ci siamo avvalsi di J. Waldron (2004), e delle considerazioni di alcuni studiosi della cosiddetta scuola austriaca di economia, e in particolare di Ludwig von Mises. 
L'uso esclusivo di un bene, caratteristico della proprietà privata, è un concetto complesso che implica:

i) che un proprietario possa usare il bene a suo piacimento sempre all'interno di una serie di usi accettabili.

ii) che gli altri individui abbiano l'obbligo di astenersi dall'uso del bene senza il permesso del proprietario.

Il proprietario ha il diritto e il potere legale di trasferire tutto l'insieme dei diritti che ha nei confronti di un bene a qualcun altro - attraverso la donazione, la vendita, o il testamento-. Dopo un'assegnazione iniziale dei beni ai proprietari, non c'è ulteriore necessità per la comunità o lo stato di occuparsi di questioni distributive: i beni circoleranno liberamente. Questo sistema può portare a risultati molto diversi: la ricchezza può essere ampiamente distribuita o può concentrarsi in poche mani.

Possiamo distinguere tre tipi di proprietà:

i) la proprietà comune

ii) la proprietà collettiva

iii) la proprietà privata.

In un sistema di proprietà comune l'accesso e l'uso delle risorse è concesso a tutti i membri della società. Un appezzamento di terra comune, per esempio, può essere usato da ogni membro della comunità - come accade nel caso di un parco aperto a tutti-. L'unica restrizione nell'uso della proprietà comune consiste nel prevenirne l'abuso, cioè nell'evitare che l'uso della risorsa comune da parte di un determinato individuo ne precluda l'uso da parte di altri membri della società.

In un sistema di proprietà collettiva la comunità nella sua totalità determina il modo in cui le risorse devono essere usate: questa determinazione viene fatta sulla base dell'interesse sociale attraverso meccanismi di decisione collettiva.

In un sistema di proprietà privata, infine, sono gli individui a possedere le risorse e possono decidere autonomamente come usarle e chi escludere dal loro uso. Come abbiamo visto in precedenza, chi possiede un determinato bene - avendolo, per 
esempio, prodotto- può anche trasferire il suo potere decisionale, e quindi i suoi diritti, sul bene vendendolo o donandolo a qualcun'altro. Quest'ultimo acquisisce gli stessi diritti che in precedenza possedeva il primo. Il proprietario, tuttavia, non ha diritto di fare qualsiasi cosa delle risorse che possiede poiché alcune sue azioni possono avere effetti anche all'esterno della sua proprietà. La proprietà privata, quindi, pur coinvolgendo solamente decisioni individuali, sembra richiedere qualche giustificazione sociale. Anche se, in generale, un sistema di proprietà privata dà il potere agli individui di prendere decisioni sull'uso di risorse scarse senza prendere in considerazione i bisogni altrui - o il bene comune-diversi autori ritengono che la società possa, almeno in determinati casi, imporre decisioni collettive sul modo di usare la proprietà privata. Esistono svariate regole che limitano la libertà individuale nell'uso delle risorse: nessuno, per esempio, può usare la propria pistola per uccidere qualcuno.

Nelle società moderne coesistono i tre tipi di proprietà sopra descritti. Alcune risorse - come le strade o i parchi- sono governate dalle regole che caratterizzano la proprietà comune, altre - come le basi militari- da quelle che caratterizzano la proprietà collettiva, e altre ancora da quelle che caratterizzano la proprietà privata. ${ }^{13}$

Come osserva de Jasay (2004, p. 57), la proprietà è una relazione tra il possessore e la cosa posseduta, tale che il proprietario è libero di usarla a suo piacimento, di concedere agli altri diritti specifici su di essa, oppure di escludere gli altri dall'accesso ad essa senza il suo consenso. Il concetto di esclusione, come si vede, è legato inseparabilmente a quello di proprietà. La proprietà, infatti, è caratterizzata dalla separazione tra proprietari e non proprietari: quando tutti possiedono una cosa, nessuno la possiede.

13 Alla natura e al ruolo della proprietà privata è stata dedicata grande attenzione da parte degli esponenti della scuola austriaca di economia, a partire da Carl Menger (1840-1921), fino a Ludwig von Mises (1883-1953) e Friedrich August von Hayek (18991992) e anche degli esponenti del cosiddetto «anarco-capitalismo», come Murray N. Rothbard (1926-1995), Anthony de Jasay (nato nel 1925) e David Friedman (nato nel 1945). 
La cosa posseduta, in genere, è una risorsa: può essere un bene tangibile, non tangibile, o la rivendicazione di un bene. Il proprietario può essere un individuo o un insieme di individui. Secondo de Jasay (2004, p 57), l'espressione "proprietà privata» è una tautologia, poiché il concetto stesso di proprietà implica la chiusura e l'esclusione. ${ }^{14}$

III

\section{LA FUNZIONE SOCIALE DELLA PROPRIETÀ PRIVATA}

«Il concetto di proprietà —osserva D. Friedman (1989, p. 21)— è fondamentale per la nostra società, probabilmente lo è per ogni società degna di questo nome. Sul piano pratico lo capisce ogni bambino sopra i tre anni. Su quello teoretico, quasi nessuno.» (D. Friedman, 1989, p. 21). Due cose, secondo D. Friedman (ivi, p. 23) rendono necessaria l'istituzione della proprietà:

i) individui diversi perseguono scopi diversi;

ii) esistono alcune risorse abbastanza scarse da non permettere a tutti di disporne liberamente.

Non possiamo avere tutti tutto ciò che vogliamo: in qualsiasi società, quindi, ci deve essere un modo per stabilire chi può usare che cosa e quando. "Il desiderio di molti di usare le stesse risorse per fini differenti è il problema centrale che rende necessaria l'istituzione della proprietà.» (D. Friedman, ivi, p. 23). Il modo più semplice di risolvere questo conflitto sarebbe l'utilizzo della forza fisica: il più forte si impossessa della risorsa che desidera. Il problema legato a questo metodo è che è troppo costoso: ogni risorsa diventerebbe oggetto di contese sanguinose. L'uso diretto della forza fisica è del tutto inefficace come soluzione al problema della limitatezza delle risorse: immaginiamo, per

14 Se non ha senso parlare di proprietà privata, non ha senso neppure parlare di "proprietà pubblica»: nessuno, infatti, viaggia in un'autostrada di proprietà statale, cioè pubblica, senza pagare il biglietto, anche se è parte di quel pubblico che possiede l'autostrada. 
esempio, cosa accadrebbe se due individui, per poter usare un'automobile, dovessero fare a botte fino a che uno solo rimane in piedi.

"La soluzione più comune è che l'uso di ogni cosa venga deciso da una persona o da un gruppo di persone organizzato da una serie di regole. Tali cose vengono chiamate proprietà.» (D. Friedman, ivi, p. 23). ${ }^{15}$ Un individuo che possiede una cosa, o risorsa, ha il potere non solo di escludere altri individui dal suo uso, ma anche di trasferirne il controllo ad altri. Attraverso lo scambio un individuo offre l'uso della sua proprietà per aiutare a realizzare gli obiettivi di un altro individuo. In cambio ha la possibilità di usare la proprietà dell'altro per realizzare i propri. In questo modo, con l'istituzione della proprietà privata, ogni individuo usa le proprie risorse per i propri fini. Nel mercato e nel commercio gli individui raggiungono i loro scopi aiutando gli altri a raggiungere i propri: attraverso tale cooperazione ciascun individuo potrà più facilmente conseguire i propri obiettivi.

Con la proprietà pubblica, le istituzioni politiche detengono beni e risorse necessari a realizzare i loro obiettivi. Poiché la funzione della politica è quella di ridurre la diversità dei fini individuali a una serie di obiettivi comuni, la proprietà pubblica cerca di imporre tali obiettivi comuni a tutti i membri della società. Lo slogan dei decisori politici sembra essere: «non chiedere come puoi perseguire ciò che ritieni giusto, ma come puoi perseguire ciò che il governo ritiene sia giusto.» (D. Friedman, ivi, p. 24). Quando la proprietà è pubblica, se un individuo richiede l'uso di quella proprietà per raggiungere i propri fini, deve sottostare alle scelte collettive attuate dal potere politico. Quando la proprietà è privata, invece, ciascuno può perseguire il proprio scopo, purché sia disposto a sopportarne il costo. Nella prospettiva di D. Friedman l'uso della proprietà privata sarà molto più oculato $\mathrm{e}$ attento di quello tipico della proprietà pubblica, perché il proprietario assume su di sé i rischi e i costi; è molto probabile, invece, che la proprietà pubblica venga gestita in modo poco

\footnotetext{
15 Corsivo nel testo.
} 
efficiente, dato che i rischi e i costi devono essere divisi fra tutti i membri della società.

Nella sua appassionata difesa della proprietà privata, von Mises (1922, pp. 55-75) sostiene che, in una società complessa, ci sono innumerevoli decisioni da prendere circa l'allocazione delle risorse. Nella maggior parte delle economie ci troviamo in presenza di innumerevoli fattori distinti di produzione cosicché sembra impossibile che decisioni efficienti riguardanti l'allocazione delle risorse vengano attuate da agenzie centralizzate - come lo statoche agiscono nel nome della comunità. Von Mises (ivi, pp. 76-90) nota che nelle società socialiste la pianificazione centrale dell'economia ha assicurato solo la paralisi economica, l'inefficienza e lo spreco. Nelle società di mercato, invece, le decisioni riguardanti le risorse e il loro impiego nel processo produttivo vengono prese in modo decentralizzato: gli individui scelgono da soli come impiegare le loro risorse cercando di massimizzare i profitti derivanti dal loro impiego. Ci si può aspettare che gli individui cerchino la massima efficienza — afferma von Mises (ibidem) solo in regime di proprietà privata perché solo in un'economia di mercato essi sono motivati dalla considerazione del profitto personale nelle loro decisioni di investimento e allocazione delle risorse. Solo in un economia di mercato, infatti, gli individui che decidono come investire le risorse sono gli stessi che guadagneranno o perderanno dagli investimenti effettuati. Si deve quindi concludere che un sistema in cui vige la proprietà privata permette l'uso più saggio ed efficiente possibile delle risorse disponibili.

IV

GIOCHI DI PROPRIETÀ. NASCITA ED EVOLUZIONE DELLA PROPRIETÀ PRIVATA NELLA PROSPETTIVA DELLA TEORIA EVOLUZIONISTICA DEI GIOCHI

Immaginiamo un gioco in cui $A$ e $B$ si contendono una risorsa - come, per esempio, un terreno- avendo a disposizione due mosse ciascuno, cioè attaccare e ritirarsi. Ipotizziamo, inoltre, che i due giocatori abbiano uguale forza e uguali capacità di 
combattere. Ciò significa che il gioco è simmetrico, cioè che le probabilità di vittoria da parte dei due giocatori sono identiche. Questo gioco viene rappresentato graficamente nella Figura 1 dove attaccare corrisponde alla mossa $i$ di $A$ e $I$ di $B$, mentre ritirarsi corrisponde alle mosse ii e II effettuabili, rispettivamente, da $A$ e $B$. Il risultato peggiore è quello in cui entrambi i giocatori attaccano, cioè (ii,II). In questo caso, infatti, si procurano entrambi ferite mortali. Leggermente migliore è il risultato in cui entrambi si ritirano; in questo caso, infatti, nessuno dei due otterrà nulla, ma almeno nessuno si farà male. Il miglior risultato per $A$ è $(i, I I)$, che si verifica quando $A$ attacca e $B$ si ritira; in tal caso, infatti, $A$ ottiene la risorsa contesa. Analogamente, il miglior risultato per $B$ è $(i i, I)$. Si può facilmente vedere che gli unici EN di questo gioco sono $(i, I I)$ e $(i i, I) .^{16}$

FIGURE 1

\section{IL GIOCO DELLA CONTESA TERRITORIALE}

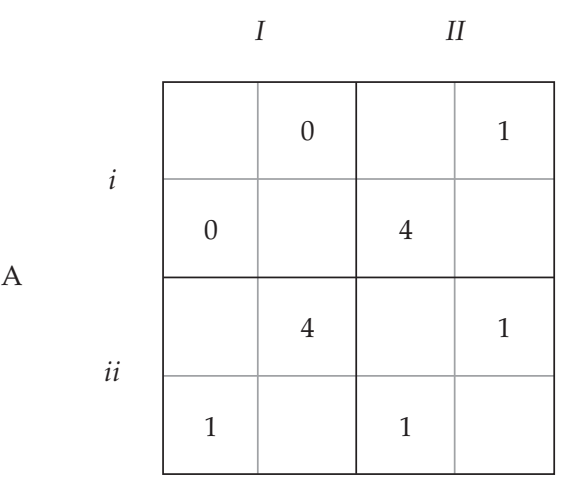

${ }^{16}$ L'equilibrio di Nash viene definito come il risultato derivante dalla combinazione di un insieme di strategie, una per ciascun giocatore, tale che, data la strategia scelta dagli avversari, nessun giocatore potrebbe migliorare la propria situazione sostituendo, unilateralmente, la strategia da lui effettivamente utilizzata con una strategia alternativa (Gibbons, 1992). 
Questo gioco, che chiameremo gioco della contesa territoriale - d'ora in poi CT - è stato studiato da vari autori che si sono occupati di teoria evoluzionistica dei giochi e di contratto sociale, quali John Maynard Smith (1982), Anthony de Jasay (1989), Richard Dawkins (1992) e Brian Skyrms (1996). Tutti questi studiosi, a partire da Maynard Smith, sono giunti a conclusioni non solo compatibili, ma anche complementari. Nelle pagine che seguono illustreremo alcuni dei risultati da essi ottenuti.

Il CT ha una struttura identica al gioco del pollo (GP), cioè a uno dei modelli di cui si è maggiormente occupata la teoria dei giochi. In questo paragrafo useremo il GP per analizzare l'origine e l'evoluzione del concetto di proprietà. ${ }^{17}$

Consideriamo ora una versione iterata del $\mathrm{CT}$ che ci permetterà di analizzare, nell'ambito della TEG, le contese territoriali. ${ }^{18}$ Immaginiamo una popolazione composta da giocatori che giocano ripetutamente fra loro e che gli incontri siano casuali. Immaginiamo, inoltre, che i giocatori si trovino a volte nel ruolo del proprietario ed altre nel ruolo dell'intruso. Supponiamo che, in uno dei turni di gioco, $A$ sia il proprietario, cioè colui che è arrivato per primo in un determinato territorio e che $B-\mathrm{l}^{\prime}$ intrusoarrivi successivamente e voglia contendere il territorio ad $A$. Consideriamo alcune possibili strategie per il CT iterato, ciascuna delle quali specifica come un giocatore deve comportarsi in ogni turno di gioco:

(S1) Se sei il proprietario, attacca; se sei l'intruso, ritirati.

(S2) Se sei il proprietario, ritirati; se sei l'intruso, attacca.

(S3) Attacca sempre.

(S4) Ritirati sempre.

17 La nascita e l'evoluzione della proprietà privata nella prospettiva della TEG sono state analizzate da diversi studiosi quali Dawkins (1976), Maynard Smith (1982), Skyrms (1996) e de Jasay (2004).

18 I giochi iterati sono costituiti da giochi ripetuti più volte nel corso di un certo periodo di tempo, che può essere determinato, oppure no, fin dall'inizio. Un gioco ripetuto, o iterato, è, in altre parole, un gioco composto dalla ripetizione di uno specifico gioco per un numero finito o indefinito di volte. 
(S1) e (S2) sono strategie condizionali, nel senso che l'azione da effettuare dipende dal ruolo del giocatore in un turno di gioco, cioè dalla circostanza che esso sia il proprietario o l'intruso. Supponiamo che in una popolazione tutti gli individui utilizzino (S1). Se nella popolazione arriva un mutante che adotta un'altra strategia — una qualsiasi altra strategia- esso scompare perché ottiene un payoff orisultato medio inferiore rispetto ai membri della popolazione: la risposta ottimale a (S1) è, infatti, (S1). Un mutante isolato, quindi, non può invadere l'intera popolazione. Ma cosa accade qualora i mutanti che adottano la stessa strategia siano più di uno? Se questi mutanti adottano (S3) o (S4), qualsiasi sia il loro numero, la media dei loro payoff sarà inferiore a quella della popolazione. Anche nel caso in cui i mutanti adottino la strategia condizionale (S2), il loro payoff sarà inferiore al payoff medio della popolazione. ${ }^{19}$ Quanto detto implica che (S1) è una SES:20 infatti, se tutti gli individui della popolazione adottano questa strategia, la popolazione non può venire invasa da mutanti che adottano una differente strategia.

Supponiamo, invece, che nella popolazione tutti gli individui adottino (S2). Poiché neppure (S2) può essere invasa da giocatori che adottano una strategia diversa, anch'essa costituisce una SES, proprio come (S1). ${ }^{21}$

Cosa accade, invece, se tutti gli individui di una popolazione adottano (S3)? Si può dimostrare che (S3) non è una SES, poiché una popolazione composta da individui che la adottano può facilmente venire invasa da un mutante che adotta una delle due strategie condizionali (S1) o (S2). Un individuo che, per esempio, adotta (S1) avrà un payoff medio superiore rispetto a quello della popolazione ed avrà, quindi, un maggiore successo riproduttivo. In questo modo i mutanti saranno sempre più numerosi fino a portare coloro che adottano (S3) all'estinzione. Le stesse considerazioni valgono anche per (S4).

19 Per una dimostrazione del fatto che queste strategie hanno un payoff medio inferiore rispetto alla popolazione si veda Skyrms (1996, pp. 65-73).

${ }^{20}$ L'acronimo SES sta ad indicare una strategia evolutivamente stabile; vedi Hargreaves (1992, pp. 169-170). Il primo ad introdurre il concetto di SES fu John Maynard Smith nel pionieristico libro Evolution and the Theory of Games (1982).

21 Skyrms (ivi, p. 67). 
Inoltre, è interessante osservare che, se tutti gli individui della popolazione adottano una delle strategie non condizionali, (S3) e (S4), allora un mutante che adotta l'altra otterrà un payoff medio superiore a quello della popolazione e il numero di mutanti che la usano crescerà. La dinamica evolutiva, in questo caso, porterà a un equilibrio misto in cui una parte della popolazione userà (S3) e l'altra (S4). Questo equilibrio misto, tuttavia, è molto inefficiente: la media dei payoff dei giocatori è inferiore non solo alla situazione in cui tutti usano una delle due SES, vale a dire (S1) o (S2), ma anche a quella in cui tutti usano (S4). Occorre anche notare che (S4) non è una SES; ciò significa che la situazione in cui tutti adottano (S4) è molto instabile; infatti, la popolazione può venire invasa facilmente da mutanti che usano (S3), fino a che non si stabilisce nuovamente la situazione inefficiente dell'equilibrio misto (Skyrms, 1996, p. 66-67). Poiché le due strategie non condizionali (S3) e (S4) non sono SES, esse possono portare a un equilibrio misto inefficiente.

Ci dobbiamo ora chiedere perché — e in che modo—l'evoluzione possa portare alla fissazione di una particolare SES e alla conseguente situazione di equilibrio.

Esistono molti modi per evitare l'inefficienza dell'equilibrio misto. Possiamo immaginare che due giocatori, prima di impegnarsi in una competizione, lancino in aria una moneta su cui siano scritti i loro nomi - uno per ogni faccia-. Un evento casuale, come l'uscita di uno dei due nomi dopo il lancio della moneta, può contribuire a rompere la simmetria in cui si trovano invischiati i giocatori e può selezionare il vincitore della contesa senza che si arrivi allo scontro fisico. Supponiamo che i due giocatori siano $A$ e $B$ : se esce $A, A$ attacca e $B$ si ritira; al contrario, se esce $B$ sarà $B$ ad attaccare ed $A$ a ritirarsi. Questa strategia viene denominata strategia randomizzata congiunta (Skyrms, ivi, p. 69). Si può mostrare che essa porta a una situazione di equilibrio: se non esce $A$, ad $A$ conviene ritirarsi perché $B$ si aspetta che $A$ si ritiri e quindi attaccherà. La strategia randomizzata congiunta porta a risultati migliori rispetto alla situazione in cui tutti usano (S4) e, quindi, migliori anche rispetto all'equilibrio misto. Un semplice evento casuale, come il lancio di una moneta, può portare alla rottura della simmetria fra i due giocatori e a una situazione di equilibrio molto più efficiente. 
Supponiamo, per esempio, che la popolazione si trovi in una situazione di equilibrio misto in cui una parte gioca (S3) e l'altra (S4). Immaginiamo poi che nasca un mutante che adotta la strategia (S5): «attacca solo se esce il tuo nome». Quando questo mutante si scontra con i giocatori che adottano una delle due strategie non condizionali (S3) e (S4), ottiene un payoff medio pari a quello della popolazione. Scontrandosi, tuttavia, con altri mutanti che adottano la stessa strategia, ottiene un payoff medio molto più alto rispetto alla popolazione. I mutanti, quindi, invaderanno tutta la popolazione. Anche i mutanti che adottano la strategia (S6), cioè «attacca solo se il tuo nome non esce» — che costituisce, per così dire, l'opposto di (S5)_ potrebbero invadere la popolazione. Qualunque tipo di mutante nasca per primo - (S5) o (S6) — esso invaderà l'intera popolazione; se invece entrambi i tipi di mutanti nascono nello stesso momento, a invadere l'intera popolazione sarà il tipo più numeroso. Una volta invasa l'intera popolazione, quella tra le strategie (S5) e (S6) che si è imposta resisterà all'invasione da parte dell'altra. Anche se non possiamo prevedere esattamente quale equilibrio si determinerà — se quello in cui tutti usano (S5) o quello in cui tutti usano (S6) - possiamo prevedere che l'evoluzione porterà allo stabilizzarsi di uno di questi due. Gli unici equilibri fortemente stabili di questo gioco sono quelli in cui tutti giocano (S5) o tutti giocano (S6).

Abbiamo visto che nel gioco della contesa territoriale, rappresentato nella figura 1 le uniche due SES sono (S1) ed (S2) - se non teniamo conto delle strategie randomizzate congiunte- Ci si può allora chiedere perché in natura si sia affermata, come risultato della dinamica evolutiva, proprio la strategia (S1), cioè «se sei il proprietario, attacca; se sei l'intruso, ritirati».

Come abbiamo detto in precedenza, un piccolo vantaggio per uno dei due giocatori può rompere la simmetria dell' equilibrio misto e portare alla stabilizzazione di una delle due SES. Sembra dunque ragionevole ipotizzare che si sia affermata la strategia (S1) poiché il giocatore che si trova per primo nel territorio conteso lo conosce meglio ed ha più energia dell'altro giocatore che ha dovuto spostarsi per raggiungerlo. La strategia (S2), 
inoltre, ha una tendenza verso l'autodistruzione: i giocatori che la adottano, infatti, cercherebbero ovviamente di essere gli intrusi in ogni scontro e, quindi, continuerebbero a muoversi, con grande dispendio di tempo ed energie; in tal modo i proprietari tenderebbero a sparire, attraverso la rinuncia al proprio ruolo. Dawkins (1976, p. 87) afferma che le strategie paradossali - come (S2) - sono stabili solo se il numero di giocatori che le adottano è ampiamente maggiore rispetto al numero di coloro che adottano SES sensate, quali (S1). ${ }^{22}$

Dawkins (1976, cap. 5) si occupa dell'aggressività nel mondo animale. In natura si può osservare che spesso gli animali combattono tra loro «in guantoni da boxe» ${ }^{23}$ : i combattimenti nel mondo animale, infatti, sembrano tornei formali, che si svolgono secondo regole come quelle del pugilato o della scherma. «Gli animali combattono con guantoni e spade senza punta, con minacce e bluff invece di uccidere sul serio. Gli atteggiamenti di resa vengono riconosciuti dai vincitori i quali si astengono quindi dal colpo o morso mortale». (ivi, p.72).

Dawkins (ivi, p. 73) sostiene che un'aperta combattività - per esempio, uccidere il rivale ogni volta che se ne ha l'opportunitàha forse alcuni vantaggi, ma anche svantaggi che non sono soltanto gli ovvi costi in tempo ed energie. In un sistema di rivalità ampio e complesso, infatti, la rimozione di un rivale dalla scena non è necessariamente un vantaggio poiché altri rivali potrebbero trarne un beneficio maggiore del nostro.

Maynard Smith (1976, p. 22) osserva che le contese tra due individui possono essere simmetriche o asimmetriche. Sono simmetriche quando i contendenti sono identici sotto tutti gli aspetti, salvo che per le strategie di lotta: in questo caso, essi possono avere strategie più o meno aggressive, ma sono ugualmente forti, ugualmente attrezzati per la lotta e hanno altrettanto da guadagnare dalla vittoria. L'ipotesi della simmetria è molto conveniente perché semplifica notevolmente il modello teorico,

22 Seguendo Dawkins (ivi, p. 86) abbiamo definito questa strategia «sensata» in base al fatto che corrisponde al «buon senso» degli esseri umani: essa, infatti, probabilmente codifica le loro pratiche diffuse di rispetto della proprietà.

23 Dawkins (ivi, p. 72). 
ma non è molto realistica. Maynard Smith (ivi, cap. 8), prende allora in considerazione le contese asimmetriche, in cui gli individui variano per dimensioni, capacità di combattimento e abilità nel giudicare le dimensioni del rivale rispetto alle proprie. Egli distingue tre tipi di asimmetrie:

i) alcune asimmetrie dipendono dal fatto che gli individui possono avere dimensioni diverse o diverso armamento;

ii) altre asimmetrie sono legate alla circostanza che gli individui possono ottenere vantaggi diversi in caso di vittoria: un maschio anziano che combatte per una femmina, per esempio, può avere meno da perdere rispetto a uno giovane poiché non gli rimane molto tempo da vivere, mentre il maschio giovane ha ancora tutta la vita riproduttiva davanti;

iii) esistono, infine, asimmetrie puramente arbitrarie che possono venire utilizzate per definire rapidamente una contesa.

Per dare un'idea di un'asimmetria arbitraria del tipo (iii) si pensi al caso in cui due contendenti decidano di dirimere una controversia lanciando in aria una moneta e scegliendo testa o croce - cioè le strategie randomizzate congiunte (S5) e (S6)-. In questo modo potrebbero risolvere la questione senza troppi costi in termini di tempo e di energie. Dawkins (ivi, p. 83-84) sostiene che la proprietà crea un'asimmetria di tipo (iii), cioè un'asimmetria arbitraria che viene usata per dirimere una contesa in modo rapido e senza eccessivi costi. Egli immagina (ivi, p. 84) che in una contesa territoriale uno dei due contendenti - che chiameremo residente- arrivi sul luogo dello scontro prima dell'altro - che chiameremo intruso-.${ }^{24}$ Inoltre, ipotizza che, in generale, non vi sia alcun vantaggio ad essere residente o intruso. Si noti che questa ipotesi è spesso irrealistica poiché, come accennato precedentemente, di solito il residente conosce meglio il territorio, o comunque non deve affrontare un faticoso spostamento per arrivare nel luogo della contesa.

Come si vede, il CT considerato da Dawkins e Maynard Smith è del tutto simile al CT analizzato da Skyrms e da noi illustrato

\footnotetext{
$24 \mathrm{Il}$ «residente» di Dawkins corrisponde al «proprietario» di Skyrms.
} 
all'inizio di questo paragrafo. Sarà quindi confortante vedere che, nell'analisi della loro versione del CT, Dawkins e Maynard Smith giungono a conclusioni non dissimili da quelle raggiunte da Skyrms.

Prendiamo in esame due strategie condizionali utilizzabili nell'affrontare il gioco: (S1) «se sei il residente, attacca; se sei l'intruso, ritirati»; (S2) «se sei il residente, ritirati; se sei l'intruso, attacca». ${ }^{25}$ Entrambe le strategie potrebbero essere stabili in una popolazione, poiché l'asimmetria tra residente e intruso è arbitraria e il primo non ha alcun reale vantaggio sul secondo; ma «quale delle due strategie evolutivamente stabili verrà adottata da una popolazione particolare dipende da quale raggiungerà per prima la maggioranza.» (Dawkins, ivi, p. 84). Infatti, non appena la maggioranza della popolazione utilizza una delle strategie condizionali (S1) e (S2), i devianti, cioè coloro che usano una strategia diversa, vengono penalizzati.

Supponiamo, per esempio, che tutti gli individui utilizzino (S1). Ciò significa che ciascuno di essi vincerà metà delle contese e ne perderà l'altra metà. Nessuno verrà mai ferito né perderà il suo tempo, poiché tutte le dispute saranno definite immediatamente per convenzione arbitraria e, più precisamente, sulla base di (S1). Immaginiamo ora l'ingresso di un mutante «ribelle» nella popolazione, cioè di un individuo che usa la strategia «attacca sempre» (S3). Esso vincerà quando il suo avversario è un intruso; tuttavia, quando l'avversario è un residente, correrà un grave rischio di restare ferito e, in media, otterrà un risultato inferiore a quello di individui che seguono le regole arbitrarie della SES (Dawkins, ivi, p. 84). Un ribelle che adotti la strategia (S2) otterrà un risultato anche peggiore: non solo sarà spesso ferito, ma raramente vincerà una contesa.

Supponiamo, invece, che la maggioranza degli individui di una popolazione usi la strategia (S2): in questo caso la loro strategia diventerebbe la norma stabile e sarebbero le deviazioni da questa ad essere penalizzate.

Nella vita reale, probabilmente, non esistono asimmetrie del tutto arbitrarie. Come abbiamo già notato, il residente può avere

${ }^{25}$ Le strategie analizzate da Dawkins corrispondono a quelle che abbiamo preso in esame all'inizio del paragrafo. 
qualche vantaggio sull'intruso perché conosce bene il territorio e non deve faticare per raggiungerlo. Esiste anche un'altra ragione per cui, delle due strategie condizionali (S1) e (S2), (S1) è di gran lunga la più frequente in natura; tale ragione consiste nella circostanza, che abbiamo rilevato in precedenza, che (S2) ha una tendenza inerente all'autodistruzione. Maynard Smith (1982, p. 102) chiama questa strategia «strategia paradossale»: se tutti gli individui di una popolazione, infatti, adottassero (S2), essi cercherebbero sempre di non essere residenti bensì di risultare intrusi in ogni incontro. Per fare ciò, dovrebbero continuare a muoversi senza sosta e senza nessun reale motivo. Oltre ai costi in termini di tempo ed energia, questo comportamento porterebbe all'estinzione dei residenti. In una popolazione in cui tutti gli individui adottano (S1), invece, la selezione naturale favorirebbe individui che cercano di essere residenti. «Per ciascun individuo questo significherebbe fermarsi in un particolare pezzo di terreno, allontanandosene il meno possibile, come se lo "difendesse". Come oggi sappiamo bene, questo comportamento si osserva comunemente in natura ed è noto come "difesa del territorio" ". (Dawkins, ivi, p. 85). La difesa territoriale può essere semplicemente una SES che si forma per l'asimmetria nel tempo di arrivo in un determinato luogo, che in genere caratterizza la relazione fra due individui e un pezzo di terra.

Una strategia paradossale, come (S2), può essere stabile perché in una popolazione formata interamente da individui che la adottano, nessuno viene ferito. Dato che la maggioranza della popolazione adotta questa strategia, un mutante che adotta una strategia sensata, come (S1), ha più probabilità di essere ferito gravemente. Le strategie paradossali possono essere stabili, ma sono poco attraenti e destinate a non durare troppo a lungo: esse, infatti, hanno un risultato medio più alto delle strategie sensate solo se gli individui che le adottano sono molto più numerosi rispetto a quelli che adottano strategie sensate.

In natura è molto difficile trovare casi in cui una popolazione adotta di norma una strategia paradossale. Un esempio di questo genere è costituito dal bizzarro comportamento del ragno sociale messicano Oecobius civitas, che viene così descritto da Burgess (1976): 
Se un ragno viene disturbato e fatto uscire dal suo rifugio, sfreccia sulla roccia e, in mancanza di un buco libero in cui nascondersi, può cercare rifugio nel nascondiglio di un altro ragno della stessa specie. Se l'altro ragno si trova nel suo buco quando l'intruso entra, non lo attacca ma sfreccia via e cerca a sua volta un nuovo rifugio. Quindi una volta che il primo ragno è stato disturbato, il processo di spostamento sequenziale da ragnatela a ragnatela può continuare per parecchi secondi, causando spesso il movimento della maggioranza dei ragni della colonia dal loro rifugio a un altro.

Skyrms (1996, p. 76) sostiene che l'essere proprietario di un bene cambia l'atteggiamento e la valutazione del bene da parte dell'individuo. Egli riprende gli studi degli economisti Kahneman, Knetsch e Thaler (1991), che passano in rassegna un'ampia letteratura sperimentale sull'argomento, riportando, fra gli altri, questo esperimento. Ad alcuni studenti di un'università americana viene data una tazza da caffè con lo stemma dell'università; successivamente viene chiesto loro a quale prezzo, compreso tra $\$ .25$ e $\$ 9.25$, sarebbero disposti a venderla. Viene inoltre chiesto ad altri studenti di offrire per la stessa tazza una somma di denaro sempre compresa tra $\$ .25$ e $\$ 9.25$. Il prezzo di riserva medio per i possessori risultò pari a $\$ 7.12$, e quello degli offerenti pari a $\$ 3.12 .{ }^{26} \mathrm{Il}$ solo fatto di essere - oppure no- il proprietario della tazza cambia, quindi, la valutazione del bene.

Anche nel mondo animale è molto diffuso questo tipo di atteggiamento nei confronti della proprietà. Il proprietario di una risorsa, come un territorio o una femmina, combatte molto più duramente per difenderla, piuttosto che per conquistarla. Per mostrare come cambia il comportamento di un animale nei confronti della proprietà, Dawkins (1976, p. 294) riprende un esperimento fatto dallo zoologo inglese Norman Davies su alcune farfalle:

26 Il prezzo di riserva di un bene è definito come il prezzo al di sotto del quale il venditore non è disposto a venderlo; analogamente, dal punto di vista del compratore, il prezzo di riserva del bene è il prezzo al di sopra del quale egli non è disposto a comprarlo. 
Davies [ ... ] notò che i singoli maschi di farfalla nel bosco di Witham, vicino a Oxford, difendevano le chiazze di sole, dalle quali le femmine erano attratte, così che queste diventavano una risorsa importante, qualcosa per cui valeva la pena di combattere. C'erano più maschi che chiazze di sole e quelli in esubero attendevano l'occasione fra le fronde degli alberi. Catturando i maschi e liberandoli uno dopo l'altro, Davies dimostrò che, prendendoli a coppie, qualunque dei due venisse rilasciato per primo in una chiazza di sole era considerato da entrambi il "proprietario", mentre qualunque maschio arrivasse secondo era considerato un «intruso». L'intruso sempre, senza eccezione, immediatamente si dichiarava sconfitto e lasciava al proprietario il possesso della chiazza di sole. In un esperimento finale decisivo, Davies riuscì ad «ingannare» due farfalle facendo «credere» a entrambe di essere le vere proprietarie. Soltanto in queste condizioni avvenne un combattimento veramente serio e prolungato.

Skyrms (1996, p. 77) si chiede come si possa spiegare la persistenza di questa valutazione apparentemente incoerente di una risorsa. Riprendendo i lavori di Maynard Smith e Dawkins, egli analizza, nel quadro della TEG, un gioco iterato in cui gli individui giocano a coppie sia nel ruolo di proprietario che in quello di intruso; il ruolo in cui si trova ciascun individuo è casuale. Skyrms (ivi, pp. 77-78) sostiene che in questo gioco l'evoluzione porta alla fissazione della strategia (S1), che all'inizio di questo paragrafo abbiamo visto essere una SES. Ciò avviene per i motivi che abbiamo analizzato in precedenza e grazie a un meccanismo che Skyrms (ibidem) definisce correlazione.

Questo meccanismo, peraltro facilmente riscontrabile nelle interazioni reali, permette agli individui che adottano strategie identiche di scontrarsi più spesso tra di loro, raggiungendo in questo modo un equilibrio correlato e ottenendo in media un payoff maggiore rispetto al resto della popolazione. La correlazione serve proprio a rompere la simmetria del gioco: permettendo, infatti, a tutti gli individui che adottano (S1) di scontrarsi più spesso tra loro, favorisce l'espansione di questa strategia all'interno della popolazione fino all'invasione della 
stessa. ${ }^{27}$ Si può quindi affermare, con Skyrms (ivi, p. 79), che «l'origine della proprietà giace nelle simmetrie spezzate».

\section{REFERENZE BIBLIOGRAFICHE}

Block, W., (1976), Defending the Undefendable, New York, Fleet Press Corporation (trad. it. Difendere l'indifendibile, Macerata, Liberilibri, 1993).

- (2003), 2003, «Overcoming difficulties in privatizing roads», Etica \& Politica / Ethics \& Politics 2, URL: «http:/ / www.units.it/ etica/2003_2/BLOCK.htm»

Burgess, J.W., (1976), «Social Spiders», Scientific American 234 (3).

Cubeddu, R,. (1997), Atlante del liberalismo, Roma, Ideazione Editrice.

- (2003), Margini del liberalismo, Soneria Mannelli, Rubettino.

Darwin, C., (1858), On the Origin of Species, London, John Murray (trad. it. L'origine della specie, Torino, Boringhieri, 1985).

Davies, N.B., (1978), «Territorial defence in the speckled wood butterfly (pararge aegeria): the resident always wins», Animal Behaviour 26.

Dawkins, R., (1976), The Selfish Gene (1989), Oxford, Oxford University Press (trad. it. Il gene egoista, Milano, Mondatori, 1992).

Epstein, J.M. e Axtell, R., (1996), Growing Artificial Societies, Whashington, The Brookings Institution.

FESTA, R., (2001), «Come evolvono le norme sociali: la prospettiva della teoria dei giochi», Biblioteca della Libertà 158.

- (2003), «Minacce e promesse: logica e metodologia della deterrenza», in La Guerra. Una riflessione interdisciplinare, a cura di G. Manganaro Favaretto, Edizioni Università di Trieste.

Friedman, D., (1989), The Machinery of Freedom, Chicago, Open Court Publishing Company (trad. it. L'ingranaggio della libertà. Guida a un capitalismo radicale, Macerata, Liberilibri, 1997).

27 Il successo riproduttivo di qualunque SES quando gioca contro se stessa garantisce a (S1) un numero sempre crescente di prole, che, col susseguirsi delle generazioni, le consentirà di invadere l'intera popolazione. 
Friedman, M., (1962), Capitalism and Freedom, Chicago, University of Chicago Press (trad. it. Capitalismo e libertà, Pordenone, Studio Tesi, 1995).

Gibbons, R., (1992), A Primer in Game Theory, Englewood Cliffs, N.J., Simon \& Schuster (trad. it. Teoria dei giochi, Bologna, Il Mulino, 1994).

Hardin, G., (1968), «The Tragedy of The Commons», Science 162, URL: «http:/ / www.dieoff.org/page95.htm»

Hargreaves Heap, S.P. et al. (1992), The Theory of Choice. A Critical Guide, Cambridge, Blackwell Publishers (trad. it. La teoria della scelta. Una guida critica, Roma, Laterza, 1996).

VON HAYEK, F.A., (1973), Law, Legislation and Liberty, vol. 3, Londra, Routledge and Kegan Paul (trad. it. Legge, legislazione e libertà, Milano, Il Saggiatore, 1994).

Holland, J.H., (1975), Adaptation in Natural and Artificial Systems, Ann Arbor, University of Michigan Press.

- (1992), «Genetic Algorithms», Scientific American 267, July (trad. it. «Algoritmi genetici», Le Scienze 289, settembre, 1992).

- (1996), Hidden Order, How Adaptation builds Complexity, Reading, MA, Persus Book.

Hume, D., (1739), A Treatise of Human Nature (trad. it. Trattato sulla natura umana, in Opere filosofiche, vol. 1, Roma-Bari, Laterza, 1993).

DE JASAY, A., (1989), Social Contract Free Ride. A Study of the Public Goods Problem, Oxford, Oxford University Press.

- (1991), Choice, Contract, Consent: a Restatement of Liberalism, London, Institute of Economic Affairs.

- (2003), «Efficiency, Opportunism and Pious Lies», Etica $\mathcal{E}$ Politica / Ethics \& Politics 2. URL: «http://www.units.it/ etica/2003_2/JASAY.htm»

- (2004), «Property and its Enemies», Philosophy 79.

KAhNemAn, D., KNETSCH, J. e Thaler, R., (1991), «The Endowment Effect, Loss Aversion and the Status Quo Bias», Journal of Economic Perspectives 5.

Leinfellner, W., (1998), «Game Theory, Sociodynamics and Cultural Evolution», in, W. Leinfellner e E. Köhler (a cura di), Game Theory, Experience and Rationality Paesi Bassi, Kluver Academic Publishers. 
LOCKE, J., (1690), The Second Treatise of Government, in Two Treatises of Government (trad. it. Trattato sul governo, Roma, Editori Riuniti, 1984).

MAYNARD SMITH, J., (1978), «The Evolution of Behavior», Scientific American 239.

- (1982), Evolution and the Theory of Games, Cambridge, Cambridge University Press.

Maynard Smith, J. e Price, G., (1973), «The Logic of Animal Conflict», Nature 146.

Menger, C., (1883), Untersuchungen über die Methode der Socialwissenschaften und der Politischen Oekonomie insbesondere, Lipsia (trad. it. Sul metodo delle scienze sociali, Macerata, Liberilibri, 1996).

von Mises, L., (1922), Die Gemeinwirtschaft: Untersuchungen über den Sozialismus, Jena (trad. it. Socialismo. Analisi economica e sociologica, Milano, Rusconi, 1990).

- (1927), Liberalismus, Jena (trad. it. Liberalismo, Soveria Mannelli, Rubettino, 1997).

- (1944), Omnipotent Government. The Rise of Total State and Total War, New Haven (trad. it. Lo stato onnipotente, Milano, Rusconi, 1995).

Mitchell, M., (1996), An Introduction to Genetic Algorithms, Cambridge, Mass., The MIT Press (trad. it. Introduzione agli algoritmi genetici, Milano, Apogeo, 1998).

Morgenstern, O., (1963), Spieltheorie und Wirtshaftswissenshaft, Vienna, R. Oldenbourg, (trad. it. Teoria dei giochi, Torino, Boringhieri,1969).

NAsH, J., (1950), «The Bargaining Problem», Econometrica 18.

- (1951), «Non-cooperative games», Annals of Mathematics 5.

- (1953), «Two persons cooperative games», Econometrica 21.

von Neumann, J. e Morgenstern, O., (1953), Theory of Games and Economic Behavior, Princeton, Princeton University Press.

Nozick, P., (1974), Anarchy, State, and Utopia, New York, Basic Books (trad. it. Anarchia, stato e utopia, Milano, Il Saggiatore, 2000).

- (1997), Socratic Puzzles, Harvard, Harvard University Press (trad. it. Puzzles socratici, Milano, Raffaello Cortina, 1999). 
Ross, D., (2003), "Game Theory», The Stanford Encyclopedia of Philosophy, Edward N. Zalta (ed.). URL:«http:/ / www.plato. stanford.edu/archives/sum2003/entries/game-theory»

Rothbard, M.N., (1965), «The Anatomy of the State», Rampart Journal I. Ristampato da N. Iannello, in La società senza stato, Soneria Mannelli, Rubettino (2004, a cura di).

- (1973), For a New Liberty. The Libertarian Manifesto, New York, Collier Books (trad. it. Per una nuova libertà. Il manifesto libertario, Macerata, Liberilibri, 1996).

- (1982), The Ethics of Liberty, Atlantic Highlands, N.J., Humanities Press Inc. (trad. it. L'etica della libertà, Macerata, Liberilibri, 1996).

Schelling, T.C., (1960), The Strategy of Conflict (1980), Oxford, Oxford University Press.

- (1978), Micromotives and Macrobehavior, New York, W.W. Norton \& Company.

- (1984), Choices and Consequences, Cambridge, Harvard University Press.

SkYRMS, B., (1994), «Darwin meets The Logic of Decision: Correlation in Evolutionary Game Theory», Philosophy of Science 61.

- (1996), Evolution of the Social Contract, Cambridge, Cambridge University Press.

- (1998), «The Shadow of the Future», in Coleman and Morris (a cura di) Rational Commitment and Social Justice. Essays for Gregory Kavka, New York, Cambridge University Press.

WALDRON, J., (2004), URL $=<$ http:/ / plato.stanford.edu/archives WeibulL, J.W. (1997), Evolutionary Game Theory, Cambridge, Mass., The MIT Press. 